\title{
EL MEDIO FÍSICO Y LOS RECURSOS NATURALES EN EL DISEÑO DE PROGRAMAS LEADER DE LA UE
}

\author{
POR \\ JOSÉ LUIS ALIER GÁNDARAS \\ ADOLFO CAZORLA MONTERO \\ IGNACIO DE LOS RÍOS CARMENADO
}

\section{Introducción}

Como consecuencia de la reforma de los Fondos Estructurales en marzo de 1991 se creó una nueva forma de acción, la iniciativa demostrativa de desarrollo rural Leader (Relaciones entre actividades de desarrollo de la economía rural), destinada a dar respuesta, mediante el desarrollo de soluciones innovadoras, a los cambios estructurales producidos en el medio rural (Comisión Comunidades Europeas, 1991).

Debido al éxito conseguido por el Leader I, gracias al cual sólo en España el número de proyectos o actuaciones financiadas llegó a 5.772, con una inversión total de 63.575 millones de pesetas, en junio de 1994 la Comisión de las Comunidades Europeas puso en marcha una segunda iniciativa de desarrollo rural denominada Leader II. Entre las novedades más importantes de esta nueva iniciativa respecto a su antecesora, cabe mencionar: la división del programa por fases (Adquisición de Capacidades, Programas Innovación Rural y Cooperación Trasnacional); el aumento de la atención hacia las necesidades de cooperación entre los distintos programas y la creación de una medida específica de ayudas destinadas a proyectos medioambientales (Comisión Comunidades Europeas, 1994).

Estudios Geográficos

Tomo LX, n. ${ }^{\circ} 236$, julio-septiembre 1999

$$
-375-
$$


Las evaluaciones de los diferentes programas LEADER I efectuados indicaron que, en gran medida, el desarrollo exitoso de los mismo depende de una buena situación de partida, de cara a identificar, junto con la población afectada, la posible utilización sostenible de los recursos naturales (Departamento de Proyectos y Planificación Rural UPM, 1996). De aquí surgió la necesidad de desarrollar la Fase de Adquisición de Capacidades como paso previo a la elaboración del Programa de Desarrollo, denominado Programa de Innovación Rural.

Sin embargo, la integración de los factores medioambientales en la selección y evaluación de los posibles proyectos subvencionables fue algo deficiente en esta primera etapa de Leader, principalmente por la dificultad intrínseca de cuantificar aspectos generalmente cualitativos. Los resultados de Leader I mostraron además que la mayoría de los proyectos utilizan recursos naturales locales, por lo que se planteó la necesidad de aumentar la atención hacia la integración de parámetros ambientales en los procesos de selección y evaluación, así como a los proyectos medioambientales (Comisión Comunidades Europeas, 1994).

Dentro de Leader II, durante el Coloquio Leader celebrado en Bruselas, se puso de manifiesto la necesidad de afianzar las relaciones entre políticas de desarrollo y políticas medio ambientales; integrar más la noción de espacio en las actividades asi como hacer extensivos a todas las acciones de desarrollo los elevados criterios de calidad en materia ambiental (Coloquio Leader II, 1998).

La posibilidad de disponer de una fase previa a la ejecución de proyectos, que sirva para detectar las posibilidades de desarrollo sostenible (Brundtland, G., 1988) evaluando con múltiples criterios los proyectos más adecuados para el desarrollo, constituye una auténtica y profunda planificación, que evita el peligro de tomas de decisiones precipitadas al subvencionar proyectos que no se engloban en las características intrínsecas de la población y del territorio (Comité de las Regiones Unión Europea, 1996).

Esta necesaria participación de los agentes locales para conseguir un desarrollo sostenible (Pezzoli, K.; 1997) ya fue expuesta en la Declaración de Principios de Río 92, en la que se resume lo que debe ser el desarrollo sostenible, y que reafirma la Declaración de la Conferencia de las Naciones Unidas sobre el Medio Humano aprobada en Estocolmo veinte años antes (UICN/PNUMA/WWF, 1991). Algunas citas de la Comisión, que insisten en esta necesidad de la participación social en el de-

$$
-376-
$$


sarrollo sostenible son: «Las mujeres desempeñan un papel fundamental en la ordenación del medio ambiente y el desarrollo. Debería movilizarse la creatividad, los ideales y el valor de los jóvenes del mundo para forjar una alianza mundial orientada a lograr un desarrollo sostenible y asegurar un mejor futuro para todos. Los pueblos y las comunidades locales, desempeñan un papel fundamental en la ordenación del medio ambiente y en el desarrollo del medio rural debido a sus conocimientos y prácticas tradicionales. Los Estados deberán reconocer y prestar el apoyo debido a su identidad, cultura e intereses y velar para que participaran efectivamente en el logro del desarrollo sostenible» (Comisión Comunidades Europeas, 1996).

Necesidad de una metodología innnovadora como soporte al Programa de Innovación Rural

El Departamento de Proyectos y Planificación Rural de la Universidad Politécnica de Madrid ha desarrollado una metodología científica de carácter innovador para cumplir los objetivos previstos de la Fase de Adquisición de Capacidades de la iniciativa Leader II: un serio diagnóstico del territorio; una motivación y formación de la población; la elaboración de una estrategia a partir de la población; para poder llegar al final de la elaboración del Programa de Innovación Rural (Observatorio Europeo de Innovación y Desarrollo Rural, 1995).

Este Programa de Innovación, que define una estrategia de desarrollo sostenible para un territorio rural determinado, se elabora de forma conjunta con la población de los agentes locales, a partir del cruce de dos líneas de información surgidas en el diagnóstico de la Comarca (Departamento de Proyectos y Planificación Rural UPM, 1997): la evaluación de las potencialidades de los recursos endógenos, y la participación social generada durante el proceso de Adquisición de Capacidades.

La metodología se elabora con un punto de referencia constante: $e l$ desarrollo rural basado en la participación de las personas afectadas (Cazorla, 1997), y se resume en dos grandes apartados (Departamento de Proyectos y Planificación Rural, 1997): A) Elaboración de un Documento base (con la definición de indicadores a nivel municipal) y emanando de los propios agentes afectados a partir de un diagnóstico participativo. B) Elaboración de un Modelo para la selección y evaluación de los proyectos que constituyen el Programa de Innovación Rural.

$$
-377-
$$




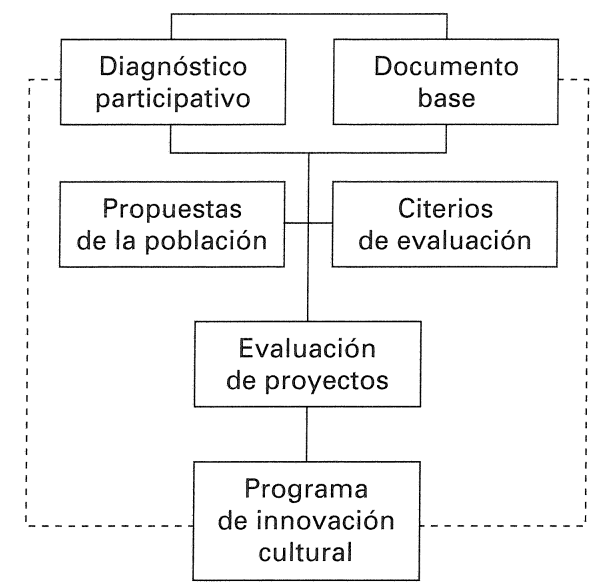

FIG. 1.-Esquema metodológico general.

El Documento base.-En el documento base se presentan las directrices sobre el modo de llevar a cabo la Iniciativa Leader II en el territorio estudiado, ahondando en todos los aspectos presentes, de cara a tener una información exhaustiva, ante la toma de decisión para la evaluación, análisis y selección de los proyectos a acometer durante la gestión del Programa de Innovación Rural. Una vez elaborado un diagnóstico completo, objetivo principal del documento base, se detectan los criterios que se incorporan al modelo para el análisis, selección y evaluación de los proyectos de forma que se garantice la participación social y la sostenibilidad de la estrategia de desarrollo. En el esquema de la página siguiente se muestran las fases interrelacionadas que componen el documento base (Fig. 2).

Para la integración de los aspectos medioambientales en el modelo de selección y evaluación de las acciones del Programa de Innovación, se parte de un profundo diagnóstico e inventario del medio físico y natural (Fase del medio fisico y los recursos naturales). Conviene tener presente que una de las principales riquezas de las zonas rurales con los valores ambientales, y que para poder conciliar medio ambiente y objetivos económicos hay que partir del territorio y los recursos locales. Por ello, de cara a generar proyectos respetuosos con el medio ambiente, enmarcados en una estrategia de desarrollo sostenible, es necesario elaborar el diagnóstico previo con esta vertiente ambiental.

$$
-378-
$$






FIG. 2.-Fases metodológicas del documento base.

La participación social.-Una estrategia de desarrollo sostenible requiere de la participación social de las comunidades rurales. El modelo de desarrollo propuesto por la Iniciativa Leader se basa, principalmente, en su carácter participativo como medio de garantizar su realización de abajo a arriba, asegurando la adecuación de los Proyectos y actuaciones a las necesidades de la población local (AEIDL, 1994). Por tanto, el diagnóstico de los recursos naturales y de los sectores productivos también deberá realizarse mediante un proceso participativo, contando con los agentes económicos de los diferentes sectores. Este diagnóstico territorial es de gran importancia en el modelo de evaluación y selección de proyectos pues facilita la relación existente entre actividad económica, territorio y recursos naturales, permitiendo, durante la gestión del Programa de Innovación, promocionar las actividades económicas respetuosas con el medio ambiente. En el gráfico adjunto se muestra el esquema metodológico propuesto para acometer esta fase participativa que permite incorporar, mediante tres fases escalonadas, la opinión de los agentes afectados de cara al diseño del Programa de Innovación Rural. La información del proceso de participación social se resume en forma de matrices DAFO, que sintetizan las Debilidades, 
Amenazas, Fortalezas y Oportunidades detectadas por la población local. Esta información se agrupa en fichas a nivel municipal y estructuradas en función de los distintos sectores, tanto productivos como no productivos.

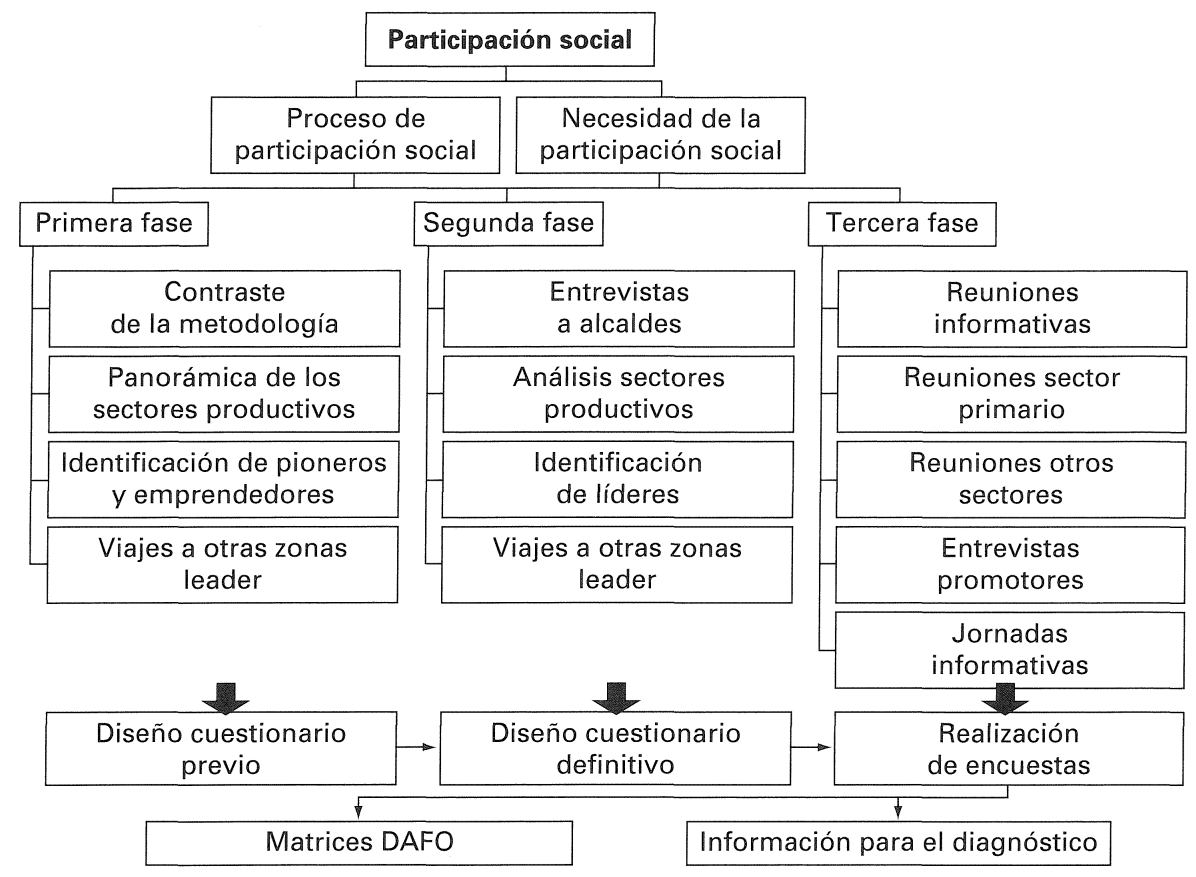

Fig. 3.-Esquema de las Fases metodológicas del documento base.

En este proceso participativo no se puede olvidar que los mayores defensores y guardianes del medioambiente son los agricultores y ganaderos, y su desaparición es el mayor desastre ecológico que se puede dar en una zona rural de elevado interés ambiental (Comisión Comunidades Europeas, 1985). Además de estudiar en profundidad el Sector primario (agrícola, ganadero y forestal), de acuerdo con las características del Leader, habrá que detenerse en los Sectores Artesanal y Turístico como fuente de proyectos ambientales para el desarrollo rural (Fuentes García, 1993). 
Transcendencia del medio físico y natural. - La sostenibilidad mantiene una estrecha relación con los recursos de los territorios, ya que el éxito de un modelo de desarrollo rural sostenible depende de la conservación de los recursos endógenos con los que cuenta, respetando los límites de los sistemas naturales (Comisión Comunidades Europeas, 1996). Por ello, la integración de los factores ambientales en la toma de decisiones referente a la evaluación de los proyectos de desarrollo no proviene únicamente de la fase del Medio Físico y los recursos naturales, aunque sí tienen una mayor importancia, sino que habrá que considerar el resto de los recursos endógenos del territorio.

Cualquier estudio del medio físico implica un acercamiento a los sistemas naturales y, por tanto, este acercamiento debe realizarse teniendo en cuenta las propiedades estructurales que caracterizan la respuesta de tales sistemas a las acciones externas, naturales o causadas por el hombre. El acercamiento debe ser sistemático, ecológico en suma, y los modelos y técnicas de análisis de los datos del inventario deben conducirse con las limitaciones sujetas al tipo de organización de los sistemas naturales, a la pauta de conexiones entre elementos, a su elasticidad o respuesta a los cambios — nunca infinita y variable en tiempo y espacio-, a su comportamiento espacial, al pattern de sus discontinuidades físicas o diversidad, a su estabilidad y variabilidad dinámica, etc. Todos estos factores determinan en gran medida sus posibilidades de supervivencia. Con la expresión del producto final del análisis de la información del inventario se termina la etapa de integración y comienzan las especificaciones que resumen el análisis y lo interpretan en función de los objetivos (Alier, Cazorla, Martínez, 1997).

Como en el caso de la participación social, la elaboración de fichas a nivel municipal que recojan toda la información ambiental facilitará el proceso posterior de evaluación y selección de los proyectos que generan un desarrollo sostenible. Los casos y problemas en que se hace necesario estudiar más a fondo el medio físico y los recursos naturales son todos aquellos en que éstos reúnen alguna de las condiciones siguientes: Aportan información relevante para el desarrollo de actividades locales, pueden sufrir modificaciones importantes por la actividad humana o poseen valores merecedores de especial protección.

$\mathrm{El}$ término medio físico es de gran amplitud y como consecuencia su estudio se aborda de muy diversas maneras, en lo que se refiere a su escala e intensidad, por lo que se hace difícil pensar en una metodología 
universal aplicable a cualquier caso. Sin embargo el número de estudios realizados y la dedicación al tema en centros académicos y de investigación permiten construir un marco metodológico sobre el que puedan apoyarse los estudios del medio físico en toda su diversidad (MOPU, 1991).

Aunque cada territorio rural posee su propio potencial y particularidades (Comisión Europea, 1996), la fase del medio físico puede llevar a seleccionar los epígrafes que se exponen en el esquema siguiente (Fig. 4).

\begin{tabular}{|c|c|}
\hline Localización & Climatología \\
\hline Geomorfología & Paisaje \\
\hline Litología & Edafología \\
\hline Hidrología & Vegetación \\
\hline Fauna & Potencial de la tierra \\
\hline Usos del suelo & Fichas municipales \\
\hline \multicolumn{2}{|c|}{ Aplicación a la evaluación } \\
\hline
\end{tabular}

FIg. 4.-Esquema de la Fase del Medio Físico.

Se trata de analizar, en primer lugar, los factores del medio físico que son condicionantes imprescindibles para la toma de decisiones en la iniciativa Leader pues aportan información relevante para el desarrollo de las actividades innovadoras ligadas al territorio, determinando su defensa ante actuaciones negativas así como su potenciación ante valores merecedores de especial protección.

Dentro de esta fase también deben ser estudiados los recursos naturales específicos de la zona de estudio, que conviene cuidar y valorar a la hora de decidir las actuaciones a llevar a cabo, pues los impactos, bien positivos o negativos, recaerán especialmente en ellos. El paisaje, la vegetación potencial, los hábitats naturales y las especies de interés comunitario, los recursos forestales, agrícolas, cinegéticos, los recursos hídricos, el potencial turístico, y el resto de los recursos endógenos de las zonas rurales, son por tanto elementos determinantes en la selección 
y evaluación de proyectos de inversión. Todos estos aspectos se deben recoger de forma ordenada en el mencionado Documento Base, de cara a las posteriores tomas de decisión.

Selección y evaluación de acciones: integración de la información.-El punto de llegada, y en donde se realiza la integración de todos los factores estudiados e inventariados (Medio Físico y Recursos Naturales, Marco Legal; Diagnóstico Socio-Económico y de los Sectores Productivos; Programas de Calidad, I + D; Participación Social y Criterios del Grupo de Acción Local), es el modelo de evaluación y selección de las acciones propuestas por la población. Es esta fase el punto crucial de cara a definir la estrategia de desarrollo sostenible - que en el caso de la iniciativa Leader se denomina Programa de Innovación Ruralya que en ella se relacionan las actividades-proyectos con los recursos del territorio y sus habitantes.

Por tanto, dentro de la gestión del Leader, durante la llamada fase de Innovación Rural, todo proyecto presentado a un Grupo de Acción Local deberá ser valorado conforme a los criterios definidos en el correspondiente Programa de Innovación Rural. Estos criterios de evaluación deben permitir valorar la adaptación de los proyectos respecto a los siguientes factores:

1. Marco legal. La revisión de la Fase Marco Legal, y las fichas que en él se incluyen, permite comprobar la adecuación de proyectos al Leader así como su posible inclusión dentro de otras líneas públicas de apoyo. 2. Diagnóstico social (generación de puestos de trabajo). El carácter de desarrollo sostenible y la proyección a largo plazo implican la consideración de las repercusiones socioeconómicas de los proyectos. Por este motivo, debe prestarse especial interés a la generación de puestos de trabajo, directos e indirectos, así como a la reducción de problemas sociales derivados de situaciones como el envejecimiento de la población local, la escasez de infraestructuras dotacionales o las necesidades formativas. 3. Medio físico y recursos naturales. Todo proyecto que pretenda ser realizado con éxito debe adecuarse a los condicionantes del medio físico y natural existente. En las Fases Medio Físico y Medio Natural se analizan todos aquellos aspectos que pudieran resultar limitantes (tipos de suelos, hidrología, climatología, etc.) para determinados proyectos, así como los principales valores medioambientales (unidades de paisaje, singularidades, vegetación, etc.) que deben ser salvaguar- 
dados. El empleo de fichas municipales — del medio físico y natural, potencialidad, usos y distribución del suelo, recursos naturales, etc.- facilita la comprobación rápida de la capacidad de acogida de cada proyecto respecto al medio físico y sus recursos. 4. Diagnóstico de los Sectores Productivos. La relación existente entre la situación de partida de cada sector económico y los proyectos que en su ámbito se planteen determina en gran medida la viabilidad de los proyectos y la situación futura del sector. Para cada proyecto se debe examinar la situación de partida del sector en el que se enmarca mediante la consulta de los apartados de análisis de los recursos del sector procedente, de cara a comprobar la adecuación del proyecto. La consulta de fichas municipales para cada sector productivo, aporta datos cuantitativos para la evaluación de la propuesta. 5. Participación social. El método Leader se basa en su carácter participativo, asegurando la adecuación de lo realizado a las necesidades de la población local. Por ello resulta adecuado evaluar las distintas propuestas que se presenten en función de la opinión y las necesidades de la población local. A tal efecto se debe comprobar la adecuación del proyecto presentado a las matrices de opinión elaboradas en la Fase de Participación Social, en las cuales se recogen sistemáticamente, mediante matrices DAFO, las principales aportaciones realizadas por la población local durante las entrevistas efectuadas. También resulta necesaria la consulta del conjunto de ideas y proyectos presentados por la Población Local, en donde se exponen las preferencias que la población local ha expresado en torno al desarrollo de su comarca. 6. Programas de Calidad, $I+D$. En el caso de que un proyecto se enmarque dentro de las líneas preferentes de desarrollo del sector, definidas desde la Administración, su viabilidad resultará mucho más evidente. Para ello se debe acudir a la Fase Programas de Calidad, $I+D$, en la que se sintetizan las líneas de apoyo a la calidad, a la investigación y al desarrollo de los productos y los sectores. 7. Criterios propios del Grupo de Acción Local. Los propios grupos LEADER pueden incluir una serie de condicionantes que sirvan para evaluar la adecuación de los proyectos al cumplimiento de sus principales objetivos, siempre que no entren en contradicción con principios generales de la propia Iniciativa. Entre estos resulta habitual la inclusión de criterios económicos (especialmente el análisis de viabilidad económica del proyecto), criterios sociales, criterios ambientales y otros criterios técnicos no dependientes del sector productivo en el que se enmarque el proyecto.

$$
-384-
$$


El equipo técnico planificador del Programa de Innovación Rural de la iniciativa Leader deberá valorar cada proyecto en función de los reseñados criterios. Esta valoración del proyecto se puede realizar en base a su inclusión en una de las siguientes cinco categorías:

A) Rechazable: el grado de incumplimiento del criterio por parte del proyecto es tal que no debe ser realizado (es decir, el proyecto queda excluido). En el caso del medio físico, una falta de capacidad de acogida o una insostenible utilización de un valioso recurso natural debe ser motivo para rechazar o replantear el proyecto. La calificación como rechazable, desde el punto de vista legal, indica la no compatibilidad del proyecto con la Iniciativa Comunitaria Leader, pero no excluye su posible realización a través de otras vías de subvención. El criterio sexto (Programa de calidad, I + D) no puede ser nunca excluyente, puesto que un proyecto puede ser especialmente interesante para la comarca y no estar dentro de las líneas preferentes de apoyo de la Administración.

B) Indiferente: el proyecto evaluado no se adapta con claridad al criterio pero tampoco lo incumple. En el caso de tratarse del primer criterio (Marco legal) conviene reconducir al proyecto hacia otras líneas de apoyo posibles.

C) Aceptable: el proyecto evaluado se adapta al criterio en cuestión, por lo que su realización sería positiva. Desde el punto de vista del medio físico presentaría una adecuación al medio aunque no producirá efectos ambientalmente positivos.

D) Especial interés: el proyecto evaluado se adapta en gran medida al criterio siendo muy conveniente su realización por su adecuación a él. Desde el punto de vista del medio físico serán proyectos de especial interés los que eliminen un problema ambiental, reduzcan la contaminación, valoricen un recurso o un territorio frágil, protejan un ecosistema valioso, disminuyan el consumo de recursos naturales, sensibilicen y formen a la población sobre cuestiones ambientales, etc.

E) Replanteable: el proyecto no se adapta al criterio al que es sometido según sus condiciones de partida o bien faltan datos para juzgar tal adaptación; pero, sin embargo, resultarían aceptables e interesantes en el caso de readaptarse a las condiciones impuestas por cada criterio o en el caso de definirse con mayor concreción. Podría ser, por ejemplo, la reubicación de una pequeña agroindustria dentro del territorio.

$$
-385-
$$


En el cuadro siguiente se resumen los criterios propuestos para la evaluación de las diferentes acciones y proyectos que pudieran proponerse junto con sus posibles calificaciones y los documentos de consulta (Cuadro I).

CuAdro I

CRITERIOS DE EVALUACIÓN DE PROYECTOS LEADER

\begin{tabular}{|c|c|c|c|}
\hline Criterio & $\begin{array}{l}\text { Documento } \\
\text { de consulta }\end{array}$ & Calificaciones & $\begin{array}{c}\text { Criterios } \\
\text { de calificación }\end{array}$ \\
\hline \multirow[t]{5}{*}{ 1. Marco legal } & \multirow[t]{5}{*}{$\begin{array}{l}\text { - Marco legal } \\
\text { - Fichas legales }\end{array}$} & Rechazable & $\begin{array}{l}\Rightarrow \text { El proyecto no entra dentro de una } \\
\text { de las medidas subvencionables por } \\
\text { LEADER }\end{array}$ \\
\hline & & Indiferente & $\begin{array}{l}\Rightarrow \text { El proyecto no es rechazable, pero } \\
\text { su adecuación no es clara }\end{array}$ \\
\hline & & Aceptable & $\Rightarrow$ Adecuación clara a la Iniciativa \\
\hline & & Especial interés & $\begin{array}{l}\Rightarrow \text { El proyecto reúne las ppales. carac- } \\
\text { terísticas exigidas, especialmente in- } \\
\text { novación y calidad }\end{array}$ \\
\hline & & Replanteable & $\begin{array}{l}\Rightarrow \text { Faltan datos o puede mejorarse la } \\
\text { adaptación }\end{array}$ \\
\hline \multirow{5}{*}{$\begin{array}{l}\text { 2. Diagnóstico } \\
\text { Social } \\
\text { (generación } \\
\text { puestos de } \\
\text { trabajo }\end{array}$} & \multirow{5}{*}{$\begin{array}{l}\text { - Diagnóstico Social } \\
\text { - Fichas } \\
\text { Socioeconómicas }\end{array}$} & Rechazable & $\Rightarrow$ Destruye puestos de trabajo \\
\hline & & Indiferente & $\begin{array}{l}\Rightarrow \text { No repercute sobre los puestos de } \\
\text { trabajo ni sobre otros aspectos so- } \\
\text { ciales }\end{array}$ \\
\hline & & Aceptable & $\begin{array}{l}\Rightarrow \text { Crea puestos de trabajo u otras me- } \\
\text { joras }\end{array}$ \\
\hline & & Especial interés & $\begin{array}{l}\Rightarrow \text { Mejora graves problemas sociales } \\
\text { existentes creando puestos de traba- } \\
\text { jo }\end{array}$ \\
\hline & & Replanteable & $\begin{array}{l}\Rightarrow \text { Faltan datos o puede mejorarse la } \\
\text { adaptación }\end{array}$ \\
\hline \multirow[t]{5}{*}{$\begin{array}{l}\text { 3edio fisico } \\
y \text { recursos } \\
\text { naturales }\end{array}$} & \multirow{5}{*}{$\begin{array}{l}\text { Estudios de } \\
\text { Capacidad e } \\
\text { Impacto } \\
\\
\text { Fichas Medio } \\
\text { Físico } \\
\text { Fichas } \\
\text { Potencialidad }\end{array}$} & Rechazable & $\begin{array}{l}\Rightarrow \text { Incompatible con las características } \\
\text { del medio físico. No permite un apro- } \\
\text { vechamiento sostenible de los recur- } \\
\text { sos naturales }\end{array}$ \\
\hline & & Indiferente & $\begin{array}{l}\Rightarrow \text { No está directamente relacionado con } \\
\text { los recursos naturales ni con el medio } \\
\text { físico }\end{array}$ \\
\hline & & Aceptable & $\begin{array}{l}\Rightarrow \text { Se integra ambientalmente y utiliza } \\
\text { los recursos naturales de forma sos- } \\
\text { tenible }\end{array}$ \\
\hline & & Especial interés & $\begin{array}{l}\Rightarrow \text { Se integra ambientalmente, utilizando } \\
\text { de forma sostenible los recursos na- } \\
\text { turales y contribuyendo a mantener o } \\
\text { restablecer algún tipo de hábitat na- } \\
\text { tural, especie u otro recurso ambien- } \\
\text { tal } \\
\end{array}$ \\
\hline & & Replanteable & $\begin{array}{l}\Rightarrow \text { Faltan datos o puede mejorarse la } \\
\text { adaptación }\end{array}$ \\
\hline
\end{tabular}


EL MEDIO FÍSICO Y LOS RECURSOS NATURALES EN EL DISEÑO...

Cuadro I (Continuación)

\begin{tabular}{|c|c|c|c|}
\hline Criterio & $\begin{array}{l}\text { Documento } \\
\text { de consulta }\end{array}$ & Calificaciones & $\begin{array}{l}\text { Criterios } \\
\text { de calificación }\end{array}$ \\
\hline \multirow[t]{5}{*}{$\begin{array}{l}\text { 4. Diagnóstico de } \\
\text { los sectores } \\
\text { deportivos }\end{array}$} & \multirow{5}{*}{$\begin{array}{l}\text { - Fase VI: } \\
\text { Diagnóstico de } \\
\text { los Sectores } \\
\text { Productivos } \\
\text { - Fichas municipales }\end{array}$} & Rechazable & $\begin{array}{l}\Rightarrow \text { El proyecto repercutiría negativa- } \\
\text { mente o es contradictorio con la si- } \\
\text { tuación de partida }\end{array}$ \\
\hline & & Indiferente & $\Rightarrow$ No influye ni es influido por el sector \\
\hline & & Aceptable & $\Rightarrow$ Se adapta a la situación \\
\hline & & Especial interés & $\begin{array}{l}\Rightarrow \text { Repercute sobre el sector positiva- } \\
\text { mente }\end{array}$ \\
\hline & & Replanteable & $\begin{array}{l}\Rightarrow \begin{array}{l}\text { Faltan datos o puede mejorar su re- } \\
\text { percusión }\end{array}\end{array}$ \\
\hline \multirow[t]{5}{*}{$\begin{array}{l}\text { 5. Participación } \\
\text { Social }\end{array}$} & \multirow{5}{*}{$\begin{array}{l}\text { - Participación } \\
\text { Social } \\
\text { - Inventario de ideas } \\
\text { y proyectos } \\
\text { aportados } \\
\text { - Matrices DAFO }\end{array}$} & Rechazable & $\begin{array}{l}\Rightarrow \begin{array}{l}\text { Es contrario a los deseos y preferen- } \\
\text { cias de la población }\end{array}\end{array}$ \\
\hline & & Indiferente & $\begin{array}{l}\Rightarrow \text { Es indiferente al sentir de la pobla- } \\
\text { ción }\end{array}$ \\
\hline & & Aceptable & $\begin{array}{l}\Rightarrow \text { Se adapta a necesidades de la pobla- } \\
\text { ción }\end{array}$ \\
\hline & & Especial interés & $\begin{array}{l}\Rightarrow \text { Existe gran interés por su implanta- } \\
\text { ción }\end{array}$ \\
\hline & & Replanteable & $\begin{array}{l}\Rightarrow \text { Faltan datos o puede mejorar su } \\
\text { adaptación }\end{array}$ \\
\hline \multirow[t]{4}{*}{$\begin{array}{l}\text { 6. Programas de } \\
\text { calidad, } I+D\end{array}$} & \multirow[t]{4}{*}{$\begin{array}{l}\text { - Programas I + D } \\
\text { - Programas calidad }\end{array}$} & Indiferente & $\begin{array}{l}\Rightarrow \text { No existe una línea específica a tal } \\
\text { efecto }\end{array}$ \\
\hline & & Aceptable & $\Rightarrow$ Existe una línea \\
\hline & & Especial interés & $\begin{array}{l}\Rightarrow \begin{array}{l}\text { Existe línea preferente de apoyo al } \\
\text { proyecto }\end{array}\end{array}$ \\
\hline & & Replanteable & $\Rightarrow$ Puede existir una línea si se readapta \\
\hline \multirow{5}{*}{$\begin{array}{l}\text { 7. Criterios del } \\
\text { GAL grupo } \\
\text { LEADER }\end{array}$} & \multirow{5}{*}{$\begin{array}{l}\text { - Información } \\
\text { del GAL }\end{array}$} & Rechazable & $\Rightarrow$ Es contrario a los deseos del GAL \\
\hline & & Indiferente & $\Rightarrow$ Es indiferente a los objetivos GAL \\
\hline & & Aceptable & $\Rightarrow$ Adaptado a los objetivos del GAL \\
\hline & & Especial interés & $\Rightarrow$ Existe gran interés por el GAL \\
\hline & & Replanteable & $\Rightarrow$ Faltan datos \\
\hline
\end{tabular}

La aplicación de estos criterios de evaluación y los parámetros para determinar la puntuación de cada proyecto se muestran a continuación: 


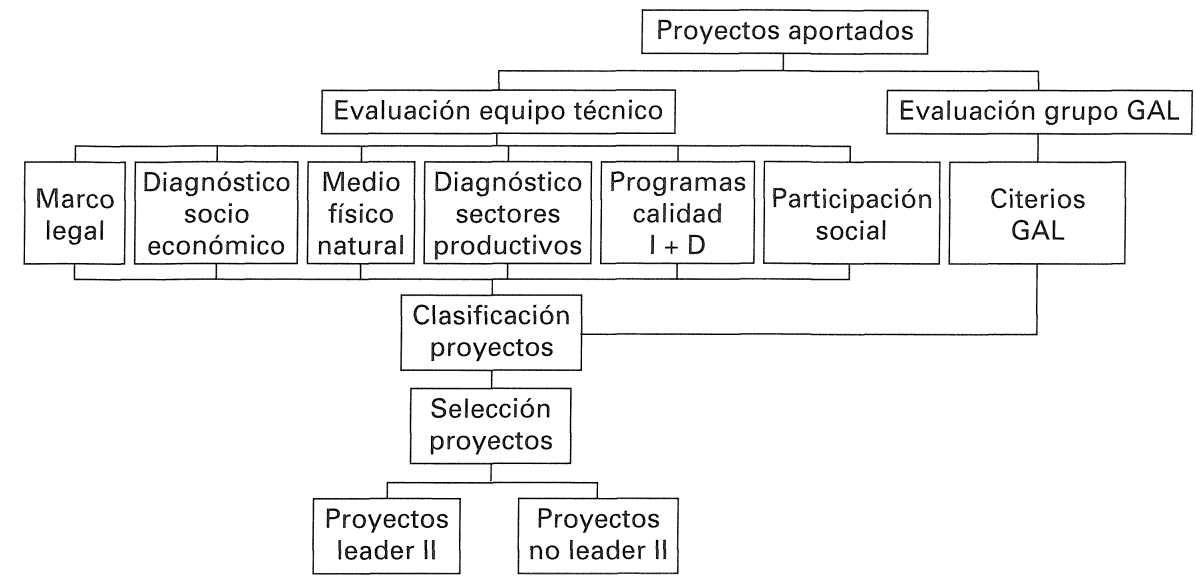

Fig. 5.-Esquema metodológico de la evaluación de proyectos.

Cuadro II

VALORACIONES SEGÚN CRITERIOS

Valoración

Evaluación por parte del equipo técnico

1. Evaluación según criterio del Marco Legal $\left(\mathrm{P}_{\mathrm{L}}\right)$

$-1,0,1,2$ y 3

2. Evaluación según criterio del Diagnóstico Socioeconómico $\left(\mathrm{P}_{\mathrm{S}}\right)$

$-1,0,1,2$ y 3

3. Evaluación según criterio del Medio Físico y recursos naturales $\left(\mathrm{P}_{\mathrm{M}}\right)$

$-1,0,1,2$ y 3

4. Evaluación según criterio del Diagnóstico S. Productivos $\left(\mathrm{P}_{\mathrm{D}}\right)$

$-1,0,1,2$ y 3

5. Evaluación según criterio de Calidad, $I+D\left(\mathrm{P}_{\mathrm{I}}\right)$

$-1,1,2$ y 3

6. Evaluación según criterio de Participación Social $\left(\mathrm{P}_{\mathrm{p}}\right)$

Evaluación por parte del Grupo de Acción Local en función de los criterios propios

1. Evaluación según criterio del Grupo de Acción Local $\left(\mathrm{P}_{\mathrm{G}}\right)$

$-1,0,1,2$ y 3

Siendo: Especial interés, 3; Aceptable, 2; Indiferente, 1; Rechazable, 0; Readaptable, -1 .

Puntuación final: $P_{F}=P_{L^{*}} P_{S^{*}} P_{M^{*}} P_{D^{*}} P_{I^{*}} P_{P^{*}} P_{G}$

La valoración definitiva de un proyecto surge del producto de las siete valoraciones parciales. De esta forma un proyecto puede ser rechazado a partir de uno sólo de los criterios, provocando una puntuación nula. Un proyecto que debe ser replanteado, desde el punto de vista de un criterio, presenta una valoración negativa y mantiene el valor abso- 
luto del resto de los criterios, a la espera de su posible modificación y transformación en positivo.

La aprobación o denegación de un proyecto se determinará en función de la discusión de dos evaluaciones, por parte del equipo técnico y por parte del Grupo de Acción Local, y la subvencionalidad de los proyectos vendrá determinada en función de la puntuación final obtenida y su rentabilidad. El factor Medio Físico y Natural es determinante, pudiendo obligar a que no se lleve a cabo una acción o facilitando al máximo su realización, mediante un mayor apoyo financiero. No obstante, para medir el grado de sostenibilidad de un proyecto determinado y su adecuación a la estrategia de desarrollo, será necesario evaluarlo respecto a todos los factores anteriormente mencionados.

\section{Un ejemplo de aplicación a la Sierra Oeste de Madrid}

La metodología descrita se ha aplicado en la elaboración del Programa de Innovación Rural de la Sierra Oeste de Madrid dentro de la fase Adquisición de Capacidades de la Iniciativa Leader II. Se trata de un Leader constituido por trece municipios del suroeste madrileño, limitando al norte con la Comunidad Autónoma de Castilla y León y al Sur con la de Castilla - La Mancha; con una superficie de $691,4 \mathrm{~km}^{2}$ y una población de 23.081 habitantes, lo que supone una densidad media de $33,4 \mathrm{hab} . / \mathrm{km}^{2}$.

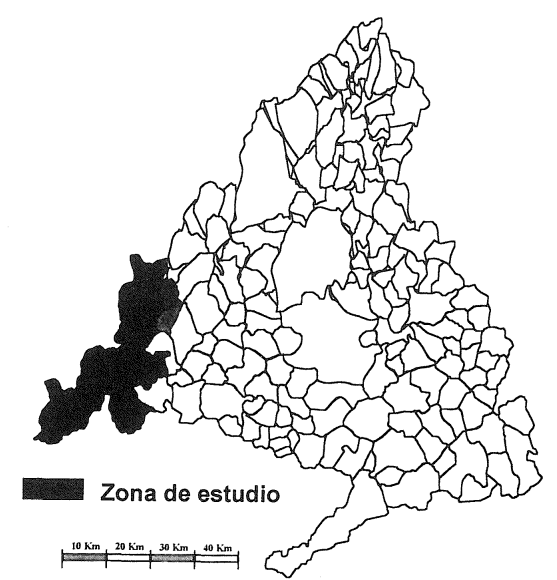

FIG. 6.-Localización del Leader II de la Sierra Oeste de Madrid. 
Durante los meses en que se elaboró la Fase de Adquisición de Capacidades en la Sierra Oeste de Madrid se desarrolló una metodología científica de carácter innovador, para cumplir los objetivos previstos en la iniciativa Leader II: un serio diagnóstico del territorio; una motivación y formación de la población; la elaboración de una estrategia a partir de la población (AEIDL, 1995). Ello conduce a la elaboración del Programa de Innovación Rural, en el que subyace una metodología participativa para la selección y evaluación de proyectos en función de los distintos criterios que definen y configuran a un territorio rural.

En la Figura 7 se representan los actores implicados en el estudio, dentro del marco de referencia del desarrollo rural participativo.

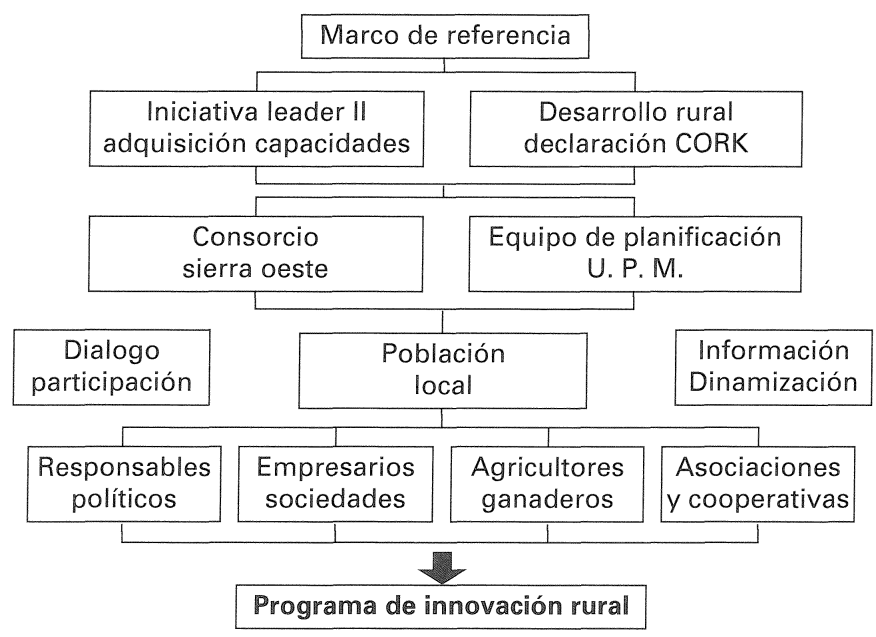

FiG. 7.-Esquema del marco de referencia y de los actores implicados.

El equipo de trabajo partía por tanto de un marco de referencia constituido por dos pilares básicos a tener en cuenta; en primer lugar, las características de la iniciativa comunitaria Leader II, y en segundo lugar, por los principios de la nueva política de desarrollo rural de la Unión Europea definidos en la Declaración de Cork (Cork, 1996). En los Cuadros III y IV se muestran los principios definidos por este marco de referencia. 
CUADro III

PRINCIPIOS DE LA INICIATIVA LEADER II

- Enfoque Plurisectorial: la iniciativa se centra en el conjunto de actividades y sectores de la economía rural.

- Programación plurianual: la segunda etapa del Leader presenta un programa vigente hasta diciembre de 1999.

- Dimensión Local o comarcal.

- Participación de la población local: los grupos de acción local y los agentes económicos de la zona son los responsables de elaborar una serie de medidas orientadas a promover el desarrollo rural así como de gestionar el Programa de Desarrollo.

- Innovación: se trata de encontrar y aplicar soluciones innovadoras respecto a las características endógenas de la zona, tanto en aspectos técnico-productivos, como ambientales y culturales.

- Efecto demostración: se respaldan las acciones que sirvan de ejemplo en su zona y con efecto multiplicador.

- Capacidad de transferencia e intercambio de conocimientos y experiencias: mediante la creación de Redes Nacionales y Europeas de Desarrollo Rural, cuya participación activa es obligatoria para todos los beneficiarios del Leader II.

- Liquidez en la Disponibilidad de Fondos: el Leader se gestiona en forma de subvención global, en donde la Administración va adelantando los Fondos comunitarios mediante anticipos a los Grupos de Acción Local.

CUADRO IV

PRINCIPIOS DE LA DECLARACIÓN DE CORK, 1996

Prioridad Rural: El desarrollo rural sostenible debe constituir una prioridad de la UE y convertirse en el principio fundamental que sustente toda política rural en el futuro inmediato y tras la ampliación. Sus objetivos deben ser: invertir éxodo rural, combatir la pobreza, fomentar el empleo y la igualdad de oportunidades, responder a la creciente demanda de calidad, salud, seguridad, desarrollo personal y ocio y mejorar el bienestar en las zonas rurales.

Enfoque integrado: La política de desarrollo rural, además de presentar una dimensión territorial clara, debe ser multidisciplinaria en su concepción y multisectorial en su aplicación; tiene que abarcar todas las zonas rurales de la Unión y respetar el principio de concentración mediante una diferente intensidad de cofinanciación de las zonas más necesitadas. Además debe basarse en un enfoque integrado que englobe dentro de un mismo marco legal e instrumental el desarrollo y reajuste de la agricultura, la diversificación económica, la gestión de los recursos naturales, la mejora de las funciones medioambientales y el fomento de la cultura, el turismo y las actividades recreativas. 


\section{CUADro IV (Continuación)}

Diversificación: La aplicación del enfoque integrado lleva a considerar a la agricultura como un elemento más del desarrollo rural. El enfoque no puede ser solo productivo, sino que habrá de fomentar una visión más amplia que permita la diversificación de las actividades hacia otros sectores económicos, sociales y ambientales. El agricultor ya no tiene la función histórica de producir, sino que también tiene una segunda función que es la de proteger el medioambiente y promover el desarrollo del mundo rural. La creación de empleos no agrícolas, la mejora de infraestructura y servicios, el aumento de los esfuerzos para mejorar el medioambiente se plantean como elementos básicos que deben formar parte de cualquier política de desarrollo rural. En esta diversificación de actividades deberán ser atendidas de forma especial las iniciativas capaces de desarrollarse por sí mismas y que emanen del sector privado y de las comunidades privadas.

Durabilidad: Las políticas deben fomentar un desarrollo rural que mantenga la calidad y la función de los espacios rurales de Europa (recursos naturales, biodiversidad e identidad cultural), de forma que el uso que hagamos hoy de ellos no menoscabe las posibles opciones de las generaciones futuras. En nuestras medidas de ámbito local debemos ser conscientes de nuestras responsabilidades globales.

Subsidiariedad: Dada la diversidad de las zonas rurales de la Unión, toda política de desarrollo rural debe respetar el principio de subsidiariedad. El principio de subsidiariedad hace referencia a la necesidad de una amplia participación de la población local, así como establecer una colaboración entre todos los niveles de responsabilidad en la política de desarrollo rural (europeo, nacional, regional y local), dando prioridad a la dimensión local. Se trata de aplicar iniciativas que emanen de la base - generadas desde abajo con un enfoque bottom-up - contando desde el inicio del proceso con la población afectada.

Simplificación: Es preciso que la política de desarrollo rural, y de forma muy especial, sus aspectos agrícolas se sometan a un proceso radical de simplificación en materia de legislación.

Programación: La aplicación de los programas de desarrollo debe basarse en procedimientos coherentes y tansparentes integrándose en un programa único de desarrollo rural propio de cada región, y en un único instrumento de desarrollo rural sostenible.

Financiación: Debe estimularse el uso de recursos financieros locales para promover proyectos de desarrollo rural. Tiene que fomentarse más el uso de la ingeniería financiera en los sistemas de crédito rural, para aprovechar mejor las sinergias entre la financiación pública y privada, reducir las limitaciones financieras de las PYMES, promover la inversión productivas y diversificar las economías rurales. 


\section{CUADRO IV (Continuación)}

Gestión: Deben impulsarse la capacidad y la eficacia administrativas regionales y locales y de los colectivos locales facilitando, allí donde ello sea necesario, asistencia técnica, formación, mejores instrumentos de comunicación, cooperación, difusión de los resultados de la investigación y de la información e intercambio de experiencias.

Evaluación e investigación: El seguimiento, la evaluación y el análisis de los resultados tendrán que reforzarse para garantizar la transparencia de los procedimientos y el bien del dinero público y para estimular la investigación y la innovación. Los interesados no sólo deben ser consultados en las tareas de concepción y aplicación, sino que además han de participar directamente en las de seguimiento y evaluación.

La zona de estudio prácticamente se incluye dentro de la ZEPA Encinares de los ríos Alberche y Cofio. Se trata de una de las siete zonas propuestas por la Administración Regional como Lugar de Importancia Comunitario (LIC), por contribuir de forma apreciable a mantener o restablecer, en un estado de conservación favorable, a algunos tipos de hábitats naturales y especies contenidas en la Directiva 92/43 CEE relativa a la conservación de los hábitats naturales y de la fauna y flora silvestres. Estos lugares LIC pueden pasar a ser designados como Zonas de Especial Conservación con el objetivo de aplicar medidas de conservación de los hábitats naturales y de las especies incluidas. El Programa de Innovación Rural debe contemplar de una forma especial estos valores ambientales de importancia comunitaria (véase Cuadro V).

En la fase del Medio Físico y los recursos naturales se analizaron todos los elementos ambientales que conviene cuidar y valorar a la hora de decidir las actuaciones a llevar a cabo sobre la comarca. Los espacios naturales de especial interés, los recursos hídricos y los hábitats naturales de interés comunitario existentes en la zona pasan a ser condicionantes ambientales, y al mismo tiempo recursos endógenos, en la evaluación de los proyectos. El objetivo es llegar a definir una estrategia de desarrollo rural integrada en la propia Zona de Especial Conservación y coherente con la Red ecológica europea, Natura 2000, compuesta por el resto de los Lugares de Importancia Comunitaria que albergan hábitats y especies prioritarias. 
CuAdro V

\section{RECURSOS NATURALES, HÁBITATS Y ESPECIES \\ DE INTERÉS COMUNITARIO INCLUIDOS \\ EN LA ZONA DE ESTUDIO}

\begin{tabular}{l|l}
\hline HABITATS NATURALES DE & - Alisedas, Bosques de ribera, Brezales orome- \\
INTERES COMUNITARIO & diterráneos; Castañares; Dehesas de encina; \\
según Directiva 92/43 CEE & Encinares; Enebrales; Fresnedas; Genista pur- \\
& gans; Gramíneas anuales; Lagos eutróficos; \\
& Matorrales esclerófilos; Pastos pioneros-roca; \\
& Prados mediterráneos y juncos; Ríos perma- \\
& nentes; Robledales; Vegetación de ribera ter- \\
& momediterránea; Vegetación de rocas silíceas; \\
& Vegetación flotante
\end{tabular}

PRINCIPALES ESPECIES DE INTERES COMUNITARIO según Directiva 92/43 CEE
- Mamíferos: Lince (especie prioritaria y en peligro de extinción); Murciélago de cueva, ratonero; Topillo de cabrera; Nutria

- Aves: Águila imperial; Águila real; Buitre negro; Buitre leonado; Halcón peregrino; Cigüeña blanca; Cigüeña negra; Cernícalo primila; Sisón; Águila culebrera; Águila calzada; Milano real y Búho real

EMBALSES CATALOGADOS según Ley 7/90 de Protección de Embalses y Zonas Húmedas de la Comunidad de Madrid

- Embalse de San Juan: con relevancia paisajística y recreativa, en los municipios de San Martín de Valdeiglesias y Pelayos de la Presa

- Embalse de Picadas: relevancia paisajística y recreativa, en los municipios de Navas del Rey y San Martín de Valdeiglesias

ESPACIOS NATURALES DE ESPECIAL INTERÉS según el Plan Forestal de la Comunidad de Madrid

- Las machotas de Zarzalejo; Meandro del Alberche en Aldea del Fresno; Dehesa del Encinar de la Parra; Castañares de Rozas de Puerto Real; Peñas de Cenicientos y Lancharrasa; Pinares de San Martín de Valdeiglesias; Peñas y Pinares de Cadalso; Pinar de Villa del Prado; Las Cabreras de San Martín de Valdeiglesias, Finca de El Hoyo; Arroyo de la Puebla; Cerro Almenara; Dehesa de Navalquejigo; Pinares de Valdemaqueda; Dehesa de Fuentelámparas; Fuentevieja 
Tras un proceso de participación abierta desarrollado a lo largo de cinco meses, durante la Adquisición de Capacidades, se obtuvieron impresiones respecto a cada uno de los sectores productivos, sobre los problemas y las potencialidades existentes en cada uno de los municipios. En el Cuadro VI siguiente se resumen para cada uno de los municipios los datos de la participación social generada: número de asistentes a las reuniones sectoriales, número de personas entrevistadas personalmente, indicador de la participación social y número de ideas de proyectos que aportaron los posibles promotores. Los 121 proyectos - propuestos por los potenciales promotores de actuaciones dentro del Programa de Innovación Rural- fueron clasificados en función de las medidas del Leader II como se refleja en el Cuadro VII.

CUADro VI

RESUMEN DE LA PARTICIPACIÓN SOCIAL

\begin{tabular}{|c|c|c|c|c|}
\hline Municipios & $\begin{array}{l}\mathrm{N}^{\circ}{ }^{\circ} \text { de } \\
\text { asis- } \\
\text { tentes }\end{array}$ & $\begin{array}{l}\text { N.de } \\
\text { encues- } \\
\text { tados }\end{array}$ & $\begin{array}{c}\text { Encues- } \\
\text { tados/ } \\
\text { asistentes }\end{array}$ & $\begin{array}{c}\mathrm{N}^{\circ} \text { de } \\
\text { pro- } \\
\text { yectos }\end{array}$ \\
\hline Cadalso de los Vidrios & 42 & 19 & $45 \%$ & 18 \\
\hline Cenicientos & 50 & 5 & $10 \%$ & 3 \\
\hline Chapinería & 23 & 10 & $43 \%$ & 4 \\
\hline Fresnedillas & 38 & 13 & $34 \%$ & 6 \\
\hline Navas del Rey & 47 & 9 & $19 \%$ & 12 \\
\hline Pelayos de la Presa & 11 & 7 & $64 \%$ & 4 \\
\hline Robledo de Chavela & 47 & 19 & $40 \%$ & 6 \\
\hline Rozas de Puerto Real & 53 & 15 & $28 \%$ & 5 \\
\hline S. M. ${ }^{a}$ de la Alameda & 47 & 19 & $40 \%$ & 9 \\
\hline S. M. de Valdeiglesias & 122 & 25 & $20 \%$ & 13 \\
\hline Valdemaqueda & 23 & 13 & $57 \%$ & 9 \\
\hline Villa del Prado & 103 & 64 & $62 \%$ & 26 \\
\hline Zarzalejo & 33 & 16 & $48 \%$ & 6 \\
\hline TOTAL & 639 & 234 & $37 \%$ & 121 \\
\hline
\end{tabular}




\title{
Cuadro VII
}

\section{CLASIFICACIÓN DE LOS PROYECTOS PROPUESTOS SEGÚN EL LEADER II}

\author{
Medida B.3.-TURISMO RURAL
}

B.3.1 Agroturismo: incorporación de las actividades agroforestales a los recursos turísticos

B.3.2 Creación de nuevas infraestructuras turísticas (alojamientos, restaurantes, esas. de servicios)

B.3.3 Diseño y puesta en marcha de nuevas actividades turísticas complementarias

B.3.4 Diseño y comercialización de productos turísticos integrados

B.3.5 Comercialización de productos turísticos locales

B.3.6 Ampliación de infraestructuras turísticas existentes

B.3.7 Promoción de los recursos e infraestructuras turísticas comarcales Medida B.4.-PEQUEÑA Y MEDIANA EMPRESA

B.4.1 Creación y desarrollo de esas. de inputs para los sectores básicos de la economía comarcal

B.4.2 Tecnificación y mejora de los sistemas de gestión y comercialización de las PYMES locales

B.4.3 PYMES con aprovechamiento de recursos locales con tecnologías respetuosas $\mathrm{m}$. ambiente

B.4.4 Servicios a las empresas locales destinados a reducir condicionantes competitivos negativos

B.4.5 Apoyo a la comercialización de productos de las empresas locales, especialmente artesanales

Medida B.5.-VALORIZACIÓN Y COMERCIALIZACIÓN DE LA PRODUCCIÓN

B.5.1 Investigación de nuevos cultivos adaptados a las condiciones edáficas y climáticas de la zona

B.5.2 Apoyo a la constitución de cooperativas, S.A.T., agrupaciones de productores, asociaciones

B.5.3 Recuperación de antiguas actividades agrarias con potencial en mercados de ptos. de calidad

B.5.4 Producción de energías alternativas aprovechando recursos endógenos

B.5.5 Estudios de mercado y de circuitos de distribución de los productos locales

B.5.6 Nuevas actividades diversificadoras para la satisfacción de nuevos productos y servicios

B.5.7 Mejoras en las estructuras de comercialización de los productos locales

B.5.8 Inversiones productivas para la mejora de la calidad y de las técnicas de transformación

B.5.9 Producciones ecológicas

B.5.10 Creación de imagen de marca y/o distintivo de calidad para los productos locales de calidad

Medida B.6.—CONSERVACIÓN Y MEJORA DEL MEDIO AMBIENTE

B.6.1 Eliminación y reciclaje de residuos

B.6.2 Revitalización de espacios naturales degradados

B.6.3 Protección y valorización de los recursos naturales y paisajísticos endógenos

B.6.4 Investigación de los recursos endógenos disponibles de cara a su aprovechamiento óptimo

B.6.5 Revalorización del patrimonio cultural de los pueblos

B.6.6 Empleo de recursos ambientales con fines turísticos de manera sostenible

B.6.7 Actuaciones destinadas a la recuperación de elementos integrantes de la cultura local 
Tras aplicar la metodología anteriormente descrita, los 121 proyectos fueron valorados de forma independiente conforme a los criterios comentados, lo que permitió diseñar un Programa de Innovación Rural coherente con los recursos de la zona y ambientalmente integrada en la futura Zona de Especial Conservación. A modo de empleo se expone en el Cuadro VIII la ficha de la valoración de uno de los proyectos propuestos por la población local.

CUADRO VIII

FICHA DE VALORACIÓN DE UN PROYECTO PROPUESTO

\begin{tabular}{|c|c|c|}
\hline \multicolumn{3}{|c|}{$\begin{array}{c}\text { PROYECTO: Diseño de sendas ecológicas en el Castañar } \\
\text { de Rozas de Puerto Real }\end{array}$} \\
\hline Criterio & Razonamiento & Valoración \\
\hline $\begin{array}{l}\text { Marco legal } \\
\qquad\left(P_{L}\right)\end{array}$ & $\begin{array}{l}\text { El proyecto reúne las características para } \\
\text { incluirse dentro de la medida subvencio- } \\
\text { nable B6 de la Iniciativa Leader de Con- } \\
\text { servación y Mejora del Medio Ambiente. } \\
\text { Existe, además un Plan de Uso y Gestión } \\
\text { de las Vías Pecuarias y de una ley de Vías } \\
\text { Pecuarias que enmarcan adecuadamente } \\
\text { el proyecto. }\end{array}$ & Especial interés (3) \\
\hline $\begin{array}{l}\text { Análisis social } \\
\text { y económico } \\
\left(P_{S}\right)\end{array}$ & $\begin{array}{l}\text { El proyecto incide de forma indirecta sobre } \\
\text { la creación de puestos de trabajo en otros } \\
\text { sectores productivos del municipio. }\end{array}$ & Aceptable (2) \\
\hline $\begin{array}{c}\text { Medio Físico } \\
\text { y recursos } \\
\text { ambientales } \\
\left(P_{M}\right)\end{array}$ & $\begin{array}{l}\text { El proyecto de carácter público, se diseña } \\
\text { precisamente para la conservación y me- } \\
\text { jora de las vías pecuarias, así como para la } \\
\text { vigilancia del hábitat natural de interés co- } \\
\text { munitario El Castañar de Rozas de Puerto } \\
\text { Real. El proyecto conserva el patrimonio } \\
\text { histórico-cultural de las vías pecuarias y } \\
\text { se integra de forma sostenible en el me- } \\
\text { dio físico del municipio. }\end{array}$ & Especial interés (3) \\
\hline $\begin{array}{c}\text { Diagnóstico de } \\
\text { los Sectores } \\
\text { Productivos } \\
\left(P_{D}\right)\end{array}$ & $\begin{array}{l}\text { El proyecto, aunque de carácter público, } \\
\text { refuerza las infraestructuras de base para } \\
\text { el desarrollo de otras actividades respe- } \\
\text { tuosas con la naturaleza, repercutiendo } \\
\text { positivamente sobre los sectores produc- } \\
\text { tivos en relación con el turismo rural y la } \\
\text { educación ambiental. }\end{array}$ & Aceptable (2) \\
\hline
\end{tabular}


CUADRO VIII (Continuación)

\begin{tabular}{c|c|c}
\hline \multicolumn{3}{c}{ PROYECTO: Diseño de sendas ecológicas en el Castañar } \\
de Rozas de Puerto Real
\end{tabular}

\section{CUADRO IX}

VALORACIÓN DE ALGUNOS DE LOS PROYECTOS DEL PROGRAMA DE INNOVACIÓN RURAL

\begin{tabular}{lcccccccc}
\hline Proyectos y actuaciones propuestas & $\mathbf{N}^{\circ} \mathbf{P r}_{\mathbf{.}}$ & $\mathbf{P}_{\mathbf{L}}$ & $\mathbf{P}_{\mathbf{S}}$ & $\mathbf{P}_{\mathbf{M}}$ & $\mathbf{P}_{\mathbf{D}}$ & $\mathbf{P}_{\mathbf{I}}$ & $\mathbf{P}_{\mathbf{P}}$ & $\mathbf{P}_{\mathbf{F}}$ \\
\hline Recuperación de la variedad «albillo» para vino & 1 & 2 & 2 & $\mathbf{2}$ & 3 & 3 & 2 & $\mathbf{1 4 4}$ \\
Valorización de la producción hortícola: Envasado y & 2 & 3 & 2 & $\mathbf{1}$ & 3 & 2 & 3 & $\mathbf{1 0 8}$ \\
Valorización de residuos: reciclado plásticos & $\mathbf{1}$ & $\mathbf{3}$ & $\mathbf{2}$ & $\mathbf{3}$ & $\mathbf{3}$ & $\mathbf{2}$ & $\mathbf{1}$ & $\mathbf{1 0 8}$ \\
Turismo ecológico: Aprovechamiento como recurso & 1 & 2 & 2 & $\mathbf{2}$ & 3 & 2 & 2 & $\mathbf{9 6}$ \\
Diversificación agraria: Introducción de espárrago & 1 & 3 & 2 & $\mathbf{2}$ & 2 & 2 & 2 & $\mathbf{9 6}$ \\
Centro de Experimentación-Transferencia de & 1 & 2 & 3 & $\mathbf{2}$ & 2 & 2 & 2 & $\mathbf{9 6}$ \\
Aprovechamiento y comercialización de recursos & $\mathbf{1}$ & $\mathbf{2}$ & $\mathbf{2}$ & $\mathbf{3}$ & $\mathbf{2}$ & $\mathbf{2}$ & $\mathbf{2}$ & $\mathbf{9 6}$ \\
Turismo ecológico: Diseño y explotación de sendas & $\mathbf{1}$ & $\mathbf{2}$ & $\mathbf{2}$ & $\mathbf{3}$ & $\mathbf{2}$ & $\mathbf{2}$ & $\mathbf{2}$ & $\mathbf{9 6}$ \\
Productos artesanos: Formación para la producción & 2 & 2 & 2 & $\mathbf{1}$ & 3 & 2 & 3 & $\mathbf{7 2}$ \\
Formación profesional vinculadas a proyectos & 1 & 3 & 2 & $\mathbf{1}$ & 3 & 2 & 2 & $\mathbf{7 2}$ \\
Elaboración de quesos artesanales & 6 & 2 & 2 & $\mathbf{1}$ & 3 & 2 & 3 & $\mathbf{7 2}$ \\
Diversificación y mejora de tecnología para la & 1 & 2 & 2 & $\mathbf{1}$ & 3 & 3 & 2 & $\mathbf{7 2}$ \\
Turismo rural: Recuperación de molinos para usos & $\mathbf{1}$ & $\mathbf{3}$ & $\mathbf{2}$ & $\mathbf{3}$ & $\mathbf{2}$ & $\mathbf{2}$ & $\mathbf{1}$ & $\mathbf{7 2}$
\end{tabular}

$N .{ }^{\circ} \mathrm{Pr} .:$ Número de propuestas presentadas para un mismo proyecto.

$P_{i} ; P_{S} ; P_{i} ; P_{0} ; P_{i}, P_{P} ; P_{F}$ : Puntuaciones respecto a los criterios de marco legal; diagnóstico socioeconómico; medio físico; diagnóstico sectores productivos; programas de calidad e I + D; participación social y puntuación final, respectivamente.

Puntuación final propuesta $P_{F}=P_{L^{*}} P_{S^{*}} P_{M^{*}} P_{D^{*}} P_{I^{*}} P_{P}$ 
Los proyectos que resultaron con una mayor valoración, y que pueden dar una idea de la mejor diversificación en la utilización del espacio, el paisaje y los recursos naturales dentro de la iniciativa Leader II en la zona de estudio, aparecen en el Cuadro IX.

Desde el punto de vista de los criterios ambientales, destacan los proyectos de valorización de residuos, turismo ecológico y aprovechamiento y valorización de recursos endógenos (agrícolas, ganaderos y forestales).

\section{Conclusiones}

Las experiencias emprendidas por los agentes locales de las zonas rurales de Europa, mediante el apoyo de Leader I y Leader II, han demostrado que la estrategia Leader es un instrumento altamente eficaz para la cohesión económica y social. Los buenos resultados han sido el motivo de que la Comisión Europea se proponga extender la metodología Leader, experimentada y validada durante ocho años, al resto de las zonas rurales. Por otra parte, para la aplicación de los Fondos Estructurales mediante los correspondientes programas operativos, para el período 2000-2006, serán necesarios programas de desarrollo que se complementan con los programas de innovación rural de la nueva iniciativa Leader.

Es por tanto necesario el continuar diseñando metodologías, que con un enfoque participativo y global, permitan integrar las exigencias de la sostenibilidad en los programas de desarrollo rural. Estas exigencias no solo provendrán de los estudios ambientales y territoriales, sino de las opiniones y conocimientos de las propias comunidades locales, desempeñando un papel fundamental en el diseño de la propia estrategia de desarrollo. Los técnicos de los programas de desarrollo rural deberán prestar por tanto una especial atención a los conocimientos y a las prácticas tradicionales, así como a la identidad y cultura de las comunidades locales.

La metodología que se propone, desarrollada en el Leader II de la Sierra Oeste de Madrid, además de integrar los factores ambientales y sociales en el Programa de Innovación Rural, se presenta como una herramienta especialmente útil en la fase de gestión de la iniciativa comunitaria de desarrollo rural, ya que está diseñada para realizar una 
evaluación multicriterio de los proyectos conforme a las características de la zona y a las singularidades del Leader. El carácter global y participativo de la metodología permite que pueda ser adaptada para diseñar otros programas de desarrollo diferentes al Leader.

\section{BIBLIOGRAFÍA}

Alier, J. L.; CAzORLA, A. y MARTínez, J. E.: Modelo informático de optimización del uso del suelo. Aplicación a tres Comunidades Autónomas. Madrid, 1996. Publicaciones del Ministerio de Agricultura, Pesca y Alimentación. Secretaría General Técnica.

BRundtland, G.: Nuestro futuro Común (Informe Brundtland). Comisión Mundial del Medio Ambiente y del Desarrollo. Naciones Unidas. Alianza Editorial. Madrid, 1988.

CAzORLA, A., et al.: Experiencias de desarrollo rural en una iniciativa Leader. Madrid, 1997. Dirección General de Agricultura y Alimentación. Consejería de Economía y Empleo. Comunidad de Madrid.

Comisión Comunidades EuRopeas: Comunicación a los Estados Miembros 91/C 73/14. Bruselas, 1991.

Comisión COMUnidades Europeas: Comunicación a los Estados Miembros. DOC 180. Bruselas, 1994.

COMisión COMUnidades EuRopeas: Informe de la Comisión. Una política de desarrollo rural. Bruselas, 1996

Comisión Comunidades EuRopeas: Informe de la Comisión sobre la aplicación del Programa Comunitario de Política y Actuación en Materia de Medio Ambiente y Desarrollo Sostenible: Hacia un Desarrollo Sostenible. Bruselas, 1996.

Departamento de Proyectos y Planificación RuRal: Determinación de Estrategias de desarrollo del sector agrícola, forestal y ganadero de la Sierra Oeste de Madrid al amparo de la Iniciativa Comunitaria LEADER II. Madrid, 1997. Universidad Politécnica de Madrid. Trabajo no publicado.

Departamento de Proyectos y Planificación Rural: Evaluación Participativa de la Iniciativa Comunitaria LEADER I en Castilla-La Mancha. Madrid, 1996. Universidad Politécnica de Madrid. Trabajo no publicado.

FuENTES GARCÍA, R.: El turismo rural en España. Especial referencia al análisis de la demanda. Madrid, 1993. Secretaría General de Turismo.

Martínez Falero, E.; CAZORla, A. et al.: Quantitative Techniques in Landscape Planning. Chicago, U.S.A., 1995. Editorial Lewis.

MOPU: Guía para la elaboración de estudios del medio físico: contenido y metodología. Madrid, 1991. Ministerio de Obras Públicas y Transportes. 3. ${ }^{a}$ edición.

ObSERvatorio Europeo de InNovación y DESARRollo RuRAL. AEIDL: Especial Coloquio Leader o Local. Leader Magazine, n. ${ }^{\circ} 16$ Bruselas, 1997.

OBSERVATORIo EuROPEO DE INNOVACIÓn y DESARROLLO RuRAL. AEIDL: La gestión local del proyecto local. Cuadernos LEADER. Bruselas, 1994.

OBSERVATORIo EuROPEO DE INNOVACIÓN y DESARROLlo RURAL. AEIDL: La constitución del proyecto de desarrollo local: la experiencia LEADER. Cuadernos LEADER. Bruselas, 1995.

ObSERVATorio EuRopeo de InNovación y Desarrollo RuRAL. AEIDL: Medio ambiente y Desarrollo Local. Leader Magazine, n. ${ }^{\circ} 6$ Bruselas, 1994.

PezzolI, K.: Sustainable Development: «A Transdisciplinary Overview of the literature». In: Environmental Planning and Management. Volume 40. Number 5. September 
1997. University of Newcastle, UK; University of New York, USA. International Periodical Publishers. CARFAX.

UICN/PNUMA/WWF: Cuidar la Tierra: Estrategia para el Futuro de la Vida. Unión Mundial para la Conservación de la Naturaleza y de los Recursos Naturales, Programa de las Naciones Unidas para el Medio Ambiente y Fondo Mundial para la Naturaleza Gland, Suiza, 1991.

RESUMEN: El Programa LEADER II es una Iniciativa Comunitaria creada para apoyar acciones innovadoras en el medio rural destinadas al desarrollo local. Durante el desarrollo del Programa LEADER I, antecesor del nuevo LEADER II, en los años 1991-94 se detectaron importantes problemas para incorporar los condicionantes medioambientales a la evaluación de proyectos subvencionables. El presente artículo muestra la metodología desarrollada en el Programa de Innovación Rural del LEADER II de la Sierra Oeste de Madrid para incorporar los factores medioambientales a la selección y evaluación de proyectos. Estos factores constituyen uno de los siete criterios - junto con el marco legal, el diagnóstico socioeconómico, el diagnóstico de los sectores productivos, la participación social, la existencia de programas de calidad e I + D y los criterios propios del Grupo de Acción Local del LEADER - desarrollados en el trabajo. El criterio del medio físico evalúa la relación entre proyecto y medio ambiente, tanto las necesidades de recursos que el proyecto presenta como los efectos de su desarrollo. De acuerdo con este criterio cada uno de los proyectos puede ser calificado con 3 puntos (especial interés), 2 puntos (aceptable), 1 punto (indiferente), 0 puntos (rechazable) ó -1 puntos (readaptable).

PALABRAS ClaVE: Desarrollo rural; iniciativa LEADER; planificación y gestión medio ambiental; evaluación de proyectos.

ABSTRACT: The LEADER II Program is a U.E. Iniciative created to support innovative actions in the rural world searching or local development. Along 1991-94 - years owning to the LEADER I Program, pattern for the new LEADER II Program- it was detected important problems to consider environmental condicionants in the evaluation of supportable proyects. This article shows a methodology developed in the Sierra Oeste (Madrid) LEADER II Program to incorporate environmental factors to the selection and evaluation of proyects. These factors constitutes one of the seven criterions - joint to the legal factors, the socioeconomic situation, the productive sector situtioin, the social opinion, the existence of quality or I + D programs and the own LEADER Sierra Oeste criterions- developed for this job. The fisic medium criterion evaluates the relation being between the proyect and the environment, either the resources needings of the proyects as the effects that its developed can produce. According to this criterion the proyects can be qualificated with 3 points - specially interesting - 2 points - acceptables - 1 point -indiferents-, 0 points — non acceptables - or -1 points - reajustables - . The article finish showing the thirteen proyects with a best qualification after apllied the seven critrions from the present methodology.

KEY WORDS: Rural development; Leader program; environmental planning and management; evaluation proyects. 